\title{
Application of an Expert System in the Management of HIV-Infected Patients
}

\author{
*Michael J. Pazzani, †Darryl See, †Edison Schroeder, and †Jeremiah Tilles \\ Departments of ${ }^{*}$ Information and Computer Sciences and $\dagger$ Medicine, University of California, Irvine, Orange, California, U.S.A.
}

\begin{abstract}
Summary: A rule-based expert system, Customized Treatment Strategies for HIV (CTSHIV), which encodes information from the literature on known drug-resistant mutations was developed. Additional rules include ranking and weighting based on antiviral activities, redundant mechanisms of action, overlapping toxicities, relative levels of drug-resistance, and proportion of drug-resistant clones in the HIV quasispecies. Plasma was obtained from HIV-infected patients and the RNA was extracted. Segments of the HIV pol gene encoding the entire protease, reverse transcriptase, and integrase proteins were amplified by reverse transcriptase-polymerase chain reaction (using a total of three primer pairs) and cloned. Sequencing was performed on five clones from each of two patients. When the patient's RNA sequencing data were entered into the expert program, and the information was downloaded directly into the CTSHIV program, the five most effective two, three, and four drug regimens coupled with an explanation for their choice were displayed for each patient. Thus, the CTSHIV system couples efficient genetic sequencing with an expert program that recommends regimens based on information in the current medical literature. It may serve as a useful tool in the design of clinical trials and in the management of HIV-infected patients. Key Words: AIDS-Expert program-PCR-Cloning-SequencingAntiretrovirals.
\end{abstract}

Many compounds have been reported to inhibit replication of the human immunodeficiency virus (HIV) both in vitro and in vivo $(1,2)$. In fact, there are already 11 antiretroviral agents licensed for use in the United States, and many more are currently undergoing preclinical and clinical evaluation. The approved agents include $2^{\prime}, 3^{\prime}$ dideoxynucleotide analog, reverse transcriptase inhibitors (azidothymidine [AZT], didanosine, dideoxycytidine, starvudine [d4T], and lamivudine [3TC]); nonnucleoside reverse transcriptase inhibitors (NNRTIs; Nevirapine, Delavirdine) and protease inhibitors (Saquinavir, Ritonavir, Indinavir, and Nelfinavir). Multiple clinical trials have demonstrated that treatment with these agents can result in improvement in markers such as CD4 count and viral luad (3-5). Other studies have

Address correspondence and reprint requests to Dr. Jeremiah Tilles, Department of Medicine, University of California, Irvine, Building 53, Route 81, 101 South City Drive, Orange, CA 92868, U.S.A.

Manuscript received August 7, 1996; accepted May 12, 1997. suggested that antiretroviral therapy can improve clinical outcomes such as mortality, occurrence of opportunistic infections, and progression to AIDS $(6,7)$. Combination therapy seems to be superior to monotherapy (8).

Unfortunately, current therapeutic regimens result in suppression but not eradication of HIV. Furthermore, antiretroviral therapy is limited by both drug toxicity and the invariable development of resistance. A variety of studies have demonstrated that resistance is associated with specific mutations in the HIV pol gene (9-12). If it were possible to directly monitor the occurrence of such mutations in the HIV RNA sequence of a given patient, specific alternative therapies might be considered before a surrogate marker (e.g., CD4 count or viral load) could be expected to even reflect a failure of the current regimen. However, detection of drug resistance may only be indicated for those patients with a rise in viral load on therapy based on studies in which continued suppression of HIV RNA was associated with a wild-type genome 
(7). Furthermore, the clinical application of sequence information in an individual patient may not currently be feasible because of the time and labor required for the sequencing process and subsequent data management.

Fortunately, computer technology can be applied to solve the problem of bulky sequence data management. Rule-based expert systems $(13,14)$ are computer programs that declaratively represent knowledge of a specialized problem and facts about a specific case, and draw inferences about the case. In the program developed for the current study (Customized Treatment Strategies for HIV [CTSHIV]), the rules encode information on drug-resistant mutations of HIV and characteristics of current antiviral agents, the facts are the sequences of the HIV genomes obtained from a specific individual, and the inference to be drawn is a set of recommended drug regimens. The knowledge of a specific problem may be represented as a set of weighted if-then rules of the form: IF < antecendent> THEN < consequence>.

For example, one such rule in CTSHIV is: IF Leucine is encoded by codon 41 of the RT portion of the pol gene, then do not use AZT (weight $=0.3$ ). The rule is derived from the literature, which reports that a methionine-toleucine mutation at codon 41 confers approximately fourfold resistance to AZT (15). The rules are weighted from 0.1 (low priority) to 1.0 (high priority) based on level of resistance or support in the literature. To draw an inference about a particular conclusion (e.g., which drugs should not be used), the expert system finds rules whose consequence matches that conclusion, and attempts to determine whether the antecedent of the rule is true. If the antecedent can be shown to be true, then the conclusion is asserted to be true. There are two ways in which an antecedent may be true. First, the antecedent may match a fact that is known. For example, in the rule just stated, this would check to see whether there is a leucine encoded by codon 41 of the RT portion of the pol gene. Second, the antecedent may match the consequence of another rule, and the antecedent of that rule can then be shown to be true.

The current study reports a process by which the protease, reverse transcriptase, and integrase segments of the HIV pol gene can be cloned using three primer sets and the sequences downloaded into an expert system that recommends multiple ranked two-, three-, and four-drug regimens based primarily on the occurrence of known mutations coding for specific drug resistance.

\section{METHODS}

\section{Cloning and Sequencing}

Blood was obtained from IU HIV-infected individuals after informed consent was obtained using a form approved by the Institutional Re- view Board at UC Irvine. All patients had CD4 counts $<500 / \mathrm{mm}^{3}$, two had never received antiretroviral therapy, and the others had received a variety of regimens. Standard reverse transcription polymerase chain reaction (RT-PCR) was performed using published primer sets for protease (16) and RT (17) and primers developed in our laboratory for integrase (upstream 5'-CAAGTAGATAAATTAG TCAGTGCTGGAATCAGG-3', downstream 5'-CCTAGCT'TTCCCTGAAACATACATATGGTGTT- $3^{\prime}$ ). PCR was performed on all 10 patients to demonstrate reproducibility of the primer sets used.

Because cloning and sequencing of PCR products is well standardized, PCR product from only two patients was sequenced. Briefly, a portion of PCR product (calculated tn give an approximate vector insert ration of 1:1) was ligated into a T/A vector (Invitrogen). Two microliters of the ligation reaction was transferred to a tube containing $50 \mu 1$ of competent Escherichia coli cells. The bacteria were transformed (18), plated onto Luria-Bertani (LB) agar plates containing $50 \mu \mathrm{g} / \mathrm{ml}$ of kanamycin, and grown at $37^{\circ} \mathrm{C}$ for $72 \mathrm{~h}$. For each plate, a single white colony was grown overnight in $5 \mathrm{ml}$ of liquid LB broth containing 50 $\mu \mathrm{g} / \mathrm{ml}$ of kanamycin. The plasmid DNA was purified and extracted using a Qiagen plasmid preparation kit (Chatsworth, CA). For the two patients, five clones per PCR product were sequenced using a dyetermination process (19) with an automated ABI sequencer. One was asymptomatic and had a CD4 count of $285 / \mathrm{mm}^{3}$ without prior antiretroviral therapy. The other patient had a history of Pneumocystis carinii pneumonia and mild wasting syndrome, had received muitiple antiretroviral regimens, and currently had a CD4 count of $55 \mathrm{cells} / \mathrm{mm}^{3}$.

\section{Development of Rules for the Expert Program}

Base substitutions coding for either in vitro and/or resistance to specific drug. (20-24) were identified in the literalure and included as rules for the expert system. The rules were weighted based on the degree of resistance. Additional rules ranked available therapies based on HIV antiviral activity, overlapping toxicities, and mechanisms of action. Currently, there are 48 rules in the program (Table 1).

\section{Development of the Expert Program}

The CTSHIV system is implemented in first-order computer learning (FOCL-1-2-3) (14), a backward chaining expert system for the Macintosh computer. The facts needed to determine which drugs to exclude are downloaded in CTSHTV. The recommendation process gnes through six distinct phases:

1. Exclusion of antiretroviral agents based on sequence information. The medical literature contains an increasing number of studies on the relationship between mutations and drug resistance.

2. Inclusion of drugs with enhanced antiretroviral activity associated with specific genetic sequences (25).

3. Exclusion of antiviral combinations with overlapping toxicities, redundant mechanisms of action, or unproven efficacy and/or safety (e.g., NNRTIs used in combination with protease inhibitors).

4. Weighting the aforementioned combinations from 0.1 (low priority) to 1.0 (high priority) based on either:

a. level of resistance conferred (phase 1; 2 -fold or $>100$-fold resistance were assigned a value of 0.1 or 1.0 , respectively; intermediate resistance was assigned a value between 0.1 and $1.0[22,23]$ ), or

b. strength of supporting evidence in the literature (phases $2-3$ $[25,26])$. 
TABLE 1. Complete set of current rules of the Customized Treatment Strategies for HIV expert program

1. AZT codon 41 RT (CTT CTC TTA CTA CTG TTG) [0.3] (15)

2. AZT codon 41 RT (CTT CTC TTA CTA CTG TTG) and codon 215 RT (TTC TAC) [1.0] (31)

3. AZT codon 67 RT (AAT AAC) and codon 70 RT (AGA AGG CGG CGC) and codon 215 RT (TTC TAC) and codon 219 RT (CAA CAG) [0.8] (32)

4. AZT codon 41 RT (CTT CTC TTA CTA CTG TTG) and codon 67 RT (AAT AAC) and codon 70 RT (AGA AGG CGG CGC) and codon 215 RT (TTC TAC) [1.0] (32)

5. AZT codon 67 RT (AAT AAC) [0.6] (33)

6. A7T codon 70 RT (AGA AGG CGG CGC) [0.6] (33)

7. AZT codon 215 RT (TTC TTT) [0.7] (33)

8. AZT codon 219 RT (CAA CAG GAA GAG) [0.1] (33)

9. ddl codon 74 RT (GTA GTG GTT) [0.4] (20)

10. (ddl ddC) codon 184 RT (GTA GTG GTT) [0.2] (34)

11. (AZT ddl d4T ddC) codon 151 RT (ATG) [1.0] (35)

12. AZT codon $210 \mathrm{RT}$ (TGG) and codon 215 ('I'IC $\mathrm{I}^{\circ} \mathrm{I}^{\mathrm{T}} \mathrm{T}$ ) [0.9] (36)

13. (AZT ddl d4T ddC) codon $69 \mathrm{RT}$ (GGA GGT GGG) [0.6] (37)

14. 3TC codon 184 RT (GTA GTG GTT) [1.0] (34)

15. 3TC codon 184 RT (ATA ATC ATT) [0.6] (34)

16. (Nevirapine Delavirdine) codon 103 RT (AAT AAC) [0.5] (38)

17. Nevirapine codon 106 RT (GCA GCT GCC) [0.9] (39)

18. Nevirapine codon 108 RT (ATA ATC ATT) [0.5] (40)

19. (Nevirapine Delavirdine) codon 181 RT (TGT TGC) [0.9] (41)

20. Nevirapine codon 181 RT (ATA ATC ATT) [0.7] (42)

21. Nevirapine codon 188 RT (TGT TGC) [0.5] (40)

22. Nevirapine codon 190 RT (GCA GCT GCC) [0.5] (39)

23. Delavirdine codon 236 RT (CTT CTC TTA CTA CTG TTG) [0.6] (43)

24. Delavirdine codon 69 RT (GGA GGT GGG) [0.2] (37)

25. Nevirapine codon 69 RT (GGA GGT GGG) [0.4] (37)

26. Ritonavir codon 8 Pro (CAA CAG AAA AAG) [0.4] (44)

27. Ritonavir codon 32 Pro (ATA ATC ATT) [0.3] (45)

28. (Ritonavir Saquinavir) codon 48 Pro (GTA GTG GTT) [0.6] (46)

29. (Ritonavir Indinavir) codon 82 Pro (GCA GCT GCC TTC TTT) [0.6] (47)

30. Ritonavir codon 10 Pro (ATA ATC ATT) [0.4] (47)

31. Ritonavir codon 54 Pro (GTA GTG GTT) [0.4] (47)
32. Indinavir codon 46 Pro (ATA ATC ATT CTT CTC TTA CTA CTG TTG) [0.6] (48)

33. Indinavir codon 82 Pro (ACA ACT ACC) [0.6] (48)

34. (Ritonavir Indinavir Saquinavir) codon 84 Pro (GTA GTG GTT) [0.6] (47)

35. Indinavir codon 32 Pro (ATA ATC ATT) [0.2] (48)

36. (Indinavir Ritonavir Nelfinavir) codon 71 Pro (GlA GIG GTT) $[0.1](47,49)$

37. Saquinavir codon 90 Pro (ATG) [0.6] (50)

38. Saquinavir codon 48 Pro (GTA GTG GTT) and codon 90 Pro (ATG) $[1.0](50)$

39. Nelfinavir codon 30 Pro (AAT AAC) [0.8] (49)

40. USE Nevirapine if codon 236 RT (CTT CTC TTA CTA CTG TTG) [0.5] (43)

41. Do not use Nelfinavir with Indinavir, Ritonavir, or Saquinavir [1.0] (51)

42. Do not use Indinavir with Ritonavir or Saquinavir [1.0] (51)

43. Do not use both Delavirdine and Nevirapine together [0.9] (8)

44. Do not use both dde and ddl together [1.0] (51)

45. Do not use either Nelfinavir, Indinavir, Ritonavir, or Saquinavir with either Nevirapine or Delavirdine [1.0] (51)

46. The expert program will receive five sequences from each patient. If all five cause a rule to fire, then multiply the weight of rule by 1.0 ; if four of five cause the rule to fire, multiply the weight by 0.8 ; if three of five cause the rule to fire, inultiply the weight by 0.6 ; if two of five cause the rule to fire, multiply the weight by 0.4 ; and if one of five cause the rule to fire, multiply the weight by 0.2 .

47. Display five two-drug regimens, five three-drug regimens, and five four-drug regimens. If more regimens are permissible, delete the following drugs in order of preference: $\mathrm{ddC}$, $\mathrm{ddl}$ Nevirapine, d4T, Saquinavir, Delavirdine, AZT, 3TC, and Nelfinavir.

48. When more than one two, three, or four drug regimens are displayed, rank each regimen by providing a value for each drug within the regimen based on the sum of the weights accumulated from the rules. Rank in descending order the regimens with the lowest total value. In case of equal value, rank in the following order: Indinavir, Ritonavir, Nelfinavir, 3TC, AZT, Delavirdine, Saquinavir, d4T, Nevirapine, ddl, ddC.

The rules were developed from information in the medical literature and integrated into the FOCL expert system shell. Most of the rules are exclusionary, based on drug-resistant mutations. For example, rule 1 is interpreted as, "Do not use AZT if the nucleotides at codon 41 of the reverse transcriptase portion of the polymerase gene are 'CCT CTC TTA CTA CTG or TTG.' " The parentheses containing the names of drugs or codons represent an "or" between the elements within them. Weights are in brackets, scaled from 0.1 (lowest priority) to 1.0 (highest priority). Exclusionary rules are weighted by the degree of drug resistance conferred. Drug interaction rules are weighted by the severity of overlapping toxicities. Disallowed drug combinations are weighted by the relative lack of supporting evidence in the literature for their use. Each weighted rule is referenced. AZT, azidothymidine; ddl, didanosine; ddC, dideoxycytidine; d4T, starvudine; 3TC, lamivudine.

5. Adjusting the weight of each conclusion by the proportion of clones causing the rule to fire.

6. Identifying and ranking combinations of candidate drugs based on antiviral activity.

\section{RESULTS}

Recommended regimens for the study patients: HIV nucleic acid was successfully amplified from all 10 patients using each of the three primer pairs. Complete sequences of five clones from two patients were downloaded into the CISHIV program and selected regimens were displayed in conjunction with explanations for their choice (Table 2). All five clones from patient 1 (CD4 count of $285 / \mathrm{mm}^{3}$, no prior antiretrovirals) had identical sequences, including 14 and 6 amino acid substitutions for RT and Pro, respectively, compared with HXB2 (Fig. 1 ). None of the changes resulted in the firing of a rule. In contrast, there was great heterogeneity among the five clones from patient 2 (CD4 count of $55 / \mathrm{mm}^{3}$, multiple antiretroviral regimens in the past). There was an average of 47 and 22 amino acid changes for RT and Pro, respectively, firing eight exclusionary rules in the expert program. 
TABLE 2. Protocols recommended for patients 1 and 2

\begin{tabular}{|c|c|c|}
\hline \multicolumn{2}{|l|}{ Protocols recommended for patient 1} & \multirow{3}{*}{$\begin{array}{l}\text { By tule } 1 \text {, AZT reduced by } 0.12 \text { because two sequences had TTG at codon } 41 \text { of the } \\
\text { RT gene. Kellam P, Boucher C, Larder BA. Fifth mutation in human } \\
\text { immunodeficiency virus type } 1 \text { reverse transcriptase contributes to the development }\end{array}$} \\
\hline The following protocols with two drugs are recommended: & & \\
\hline Indinavir 3TC & 0.0 & \\
\hline Indinavir AZT & 0.0 & of high-level resistance to zidovudine. Proc Natl Acad Sci USA 1992:89:1934-8. \\
\hline Ritonavir 3TC & 0.0 & By rule 28 , Ritonavir reduced by 0.24 because two sequences had GTG at \\
\hline Ritonavir AZT & 0.0 & 0.6 codon 48 of the Pro gene. Boucher $C$. Rational approaches to resistance: \\
\hline $3 \mathrm{TC}$ AZT & 0.0 & Using saquinavir. AIDS 1996;10(suppl 1):S15-9. \\
\hline \multicolumn{3}{|l|}{ The following protocols with three drugs are recommended: } \\
\hline Indinavir 3TC AZT & 0.0 & By rule 5 , AZT reduced by 0.6 because five sequences had AAC at codon 67 \\
\hline Ritonavir 3TC AZT & 0.0 & of the RT gene. Caliendo A, Savara A, An D, DeVore K, Kaplan J, \\
\hline Ritonavir 3TC Saquinavir & 0.0 & D'Aquila $R$. Effects of zidovudine-selected human immunodeficiency virus \\
\hline Ritonavir AZT Saquinavir & 0.0 & type 1 reverse transcriptase amino acid substitutions on processive DNA \\
\hline 3TC AZT Delavirdine & 0.0 & synthesis and viral replication. I Virol $1996 ; 70: 2146-53$. \\
\hline \multicolumn{3}{|l|}{ The following protocols with four drugs are recommended: } \\
\hline Indinavir 3TC AZT D4T & 0.0 & By rule 34 , Indinavir, Saquinavir, Ritonavir reduced by 0.6 because five sequences \\
\hline Rotonavir 3TC AZT Saquinavir & 0.0 & had GTG at codon 84 of the Pro gene. Schmidt J, Ruiz L, Clotet B, et al. \\
\hline Ritonavir $3 \mathrm{IC}$ ACI D $4 \mathrm{~T}$ & 0.0 & Resistance-related mutations in the HIV-1 protease gene of patients treated \\
\hline Ritonavir 3TC Saquinavir D4T & 0.0 & for 1 year with the protease inhibitor ritonavir (ABT-538). AIDS 1996;10:995-9. \\
\hline Ritonavir AZT Saquinavir D4T & 0.0 & \\
\hline \multirow{2}{*}{\multicolumn{2}{|c|}{ Protocols recommended for patient 2}} & By rule 38 , Saquinavir reduced by 0.4 because two sequences had $\mathrm{G} / \mathrm{G}$ at codon 48 \\
\hline & & of the Pro gene and ATG at codon 90 of the Pro gene. Jacobsen $\mathrm{H}$, Haenggi M. \\
\hline \multicolumn{2}{|l|}{ The following protocols with two drugs are recommended: } & Ott M, Duncan I, Andreoni M, Vella S, Mous J. Reduced sensitivity to saquinavir: \\
\hline 3TC Delavirdine & 0.0 & An update on genotyping from phase I/II trials. Antiviral Res $1996 ; 29: 95-7$. \\
\hline $3 \mathrm{TC} \mathrm{d} 4 \mathrm{~T}$ & 0.0 & \\
\hline 3TC Nevirapine & 0.0 & By rule 9 , ddl reduced by 0.4 because five sequences had GTA at codon 74 \\
\hline Delavirdine $\mathrm{d} 4 \mathrm{~T}$ & 0.0 & of the RT gene. St Clair M, Martin $\mathbf{J}$, Tudor W. Resistance to ddl and \\
\hline $3 \mathrm{TC} \mathrm{ddC}$ & 0.0 & sensitivity to AZT induced by a mutation in HIV-1 reverse transcriptase. \\
\hline \multicolumn{2}{|l|}{ The following protocols with three drugs are recommended: } & Science 1991;253:1557-9. \\
\hline 3TC Delavirdine $\mathrm{d} 4 \mathrm{~T}$ & 0.0 & \\
\hline 3TC d4T Nevirapine & 0.0 & By rule 6, AZT reduced by 0.6 because five sequences had AGA at codon \\
\hline 3TC Delavirdine ddC & 0.0 & 70 of the RT gene. Caliendo A, Savara A, An D, DeVore K, Kaplan J, \\
\hline $3 \mathrm{TC}$ d4T ddC & 0.0 & D'Aquila R. Effects of zidovudine-sclected human immunodeficiency virus \\
\hline $3 T C$ Nevirapine ddC & 0.0 & type 1 reverse transcriptase amino acid substitutions on processive DNA \\
\hline \multicolumn{2}{|l|}{ The following protocols with four drugs are recommended: } & synthesis and viral replication. $J$ Virol $1996 ; 70 ; 2146-53$. \\
\hline 3TC Delavirdine $\mathrm{d} 1 \mathrm{~T}$ ddC & 0.0 & \\
\hline $3 \mathrm{TC}$ d4T Nevirapine ddC & 0.0 & By rule 37 , Saquinavir reduced by 0.36 because three sequences had ATG \\
\hline 3TC Delavirdine $\mathrm{d} 4 \mathrm{~T}$ ddl & 0.4 & at codon 90 of the Pro gene. Jacobsen H, Haenggi M, Ott M, Duncan I, \\
\hline $3 \mathrm{TC}$ d4T Nevirapine ddl & 0.4 & Andreoni $M$, Vella $S$, Mous J. Reduced sensitivity to saquinavir An update \\
\hline $3 \mathrm{TC}$ d4T ddC Indinavir & 0.6 & on genotyping from phase U/I trials. Antiviral Res $1996 ; 29: 95-7$. \\
\hline
\end{tabular}

\section{DISCUSSION}

Antiretroviral therapy is useful in slowing down the progression of HIV infection. However, all therapeutic regimens studied to date eventually fail, resulting in clinical deterioration of the patient and worsening of surrogate markers such as a rise in CD4 count, an increase in viral load, or both. The current standard of practice indicates a change in therapy when this occurs. However, guidelines as to specific changes in antiretrovirals is lacking, and the possibility of appropriately changing therapy for specific mutants before surrogate marker deterioration is possible. The time to onset of clinically important mutations is highly variable. Cross-resistance occurs between agents in the same class or with similar mechanisms of action (21). Finally, drug-specific resistance mutations also occur in wild-type strains (22). Thus, basing changes in therapy on drug sensitivity would seem to have merit. The results of the current study demonstrate the feasibility of such an approach using an expert program to recommend treatment regimens. The program also encodes information about level of drug resistance, overlapping toxicities, and other fac- tors. Learning algorithms such as FOCL (14) can be applied to the program to modify it based on outcome measures from patients such as CD4 count, viral load, and progression to AIDS. Old rules can be reweighted or eliminated. New rules can be added based on information from the literature or the development of new antiretroviral agents.

The HIV strains in an individual patient are not genomically homogenous. The current system allows detection of a resistant mutation if present in at least one of five clones. If detection of crucial mutations in a patient found less frequently than $20 \%$ is later thought to be important, hybridization techniques (27) could be applied to capture those present in as few as 1 of 20 strains.

The CTSHIV system uses primers from conserved regions, yielding detectable PCR products for each of the 10 patients tested. Furthermore, it contains an expert system to effectively manage the sequence data and recommend specific therapeutic regimens based on genetic sequences and information from the medical literature. Nucleotide sequences could be aligned using software programs (28), reducing the chance of downloading indeterminate foci. However, the current system incorpo- 
position 11

21

31

41

51

61

71

HXB2 CCTCAGGTCA CTCTTTGGCA ACGACCCCTC GTCACAATAA AGATAGGGGG GCAACTAAAG GAAGCTCTAT TAGATACAGG

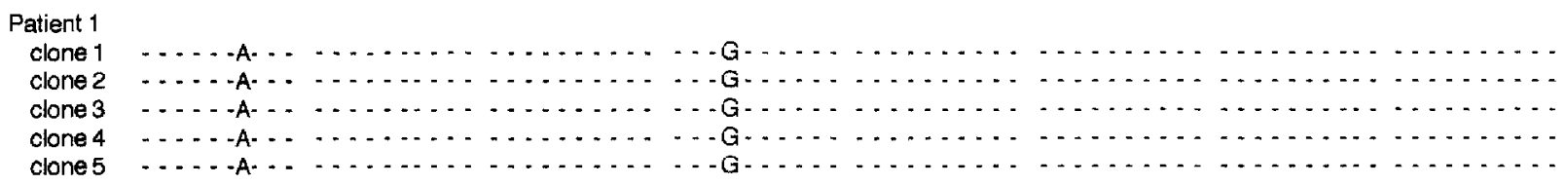

Patient 2

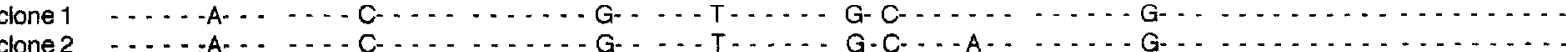

clone 3

clone 4

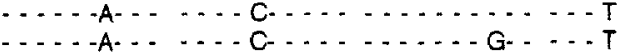

clone 5

81

91

101

111

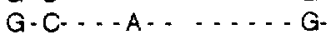

$-C-\ldots A-\ldots \ldots$ G. $\ldots \ldots \ldots \ldots$

G.C. . A $\ldots \ldots \ldots$ G. . . . . . . . .

121

131

141

151

$\mathrm{HXB2}$

AGCAGATGAT ACAGTATTAG AAGAAATGAG TTTGCCAGGA AGATGGAAAC CAAAAATGAT AGGGGGAATT GGAGGTTTTA

Patient 1

clone 1

clone 2

clone 3

clone 4

clone 5

Patient 2

clone 1

clone 2

done 3

clone 4

clone 5

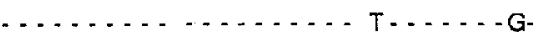

161

171

181

191

201

211

221

231

$\mathrm{HXB2}$

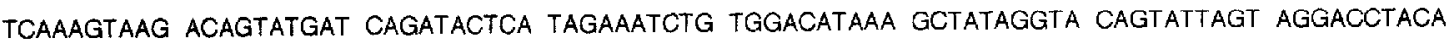

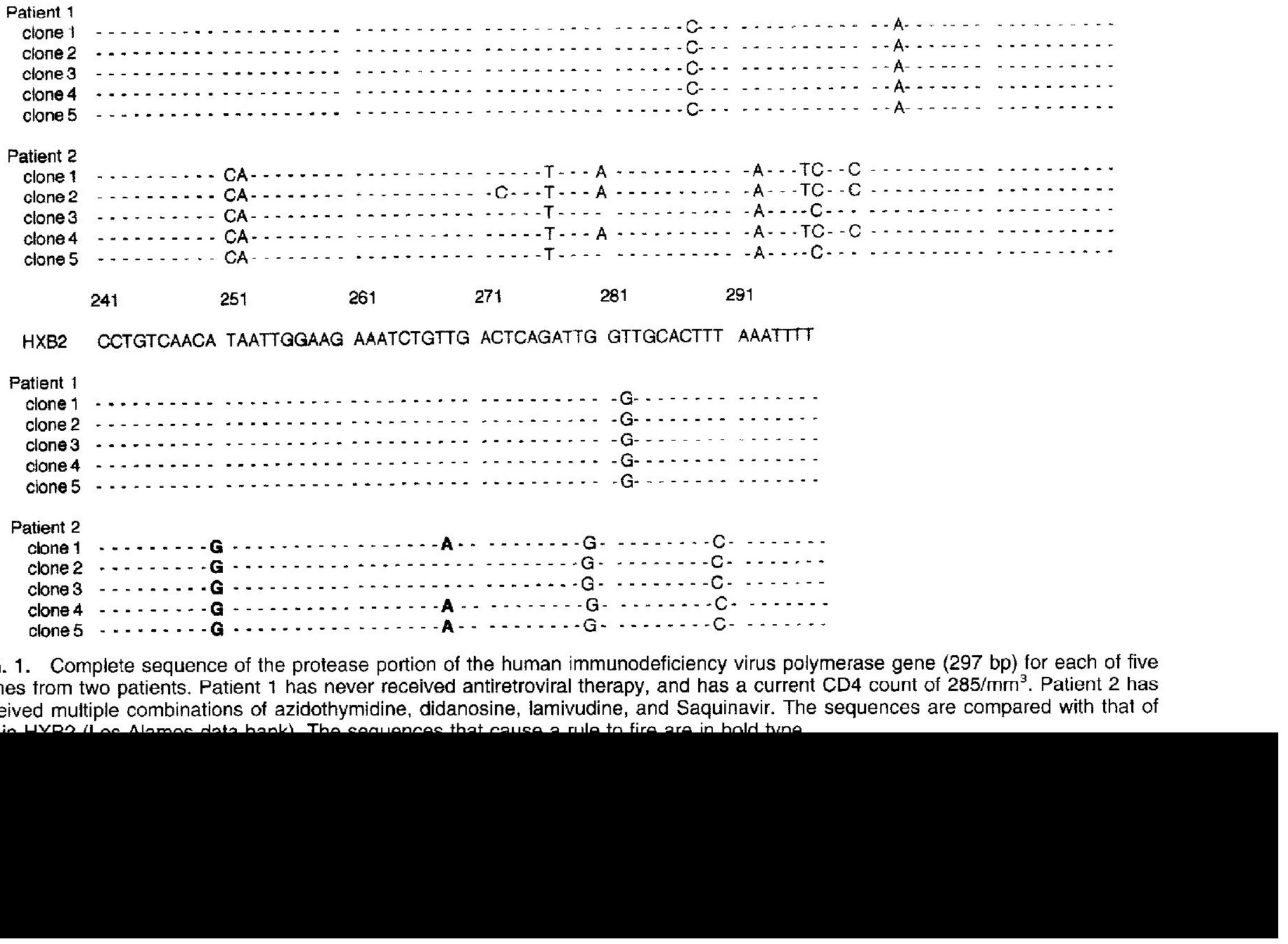

FIG. 1. Complete sequence of the protease portion of the human immunodeficiency virus polymerase gene (297 bp) for each of five clones trom two patients. Patient 1 has never received antiretroviral therapy, and has a current CD4 count of $285 / m m{ }^{3}$. Patient 2 has received multiple combinations of azidothymidine, didanosine, lamivudine, and Saquinavir. The sequences are compared with that of 
rates sequencing of multiple clones. Although our preliminary studies suggested that such a process both reduces the display of indeterminate residues and enhances the opportunity to identify drug-resistant strains representing a small proportion of the total number of quasispecies in an individual patient compared with sequencing the heterogenous mixture of strains, it also adds significantly to the time and cost. In this pilot study, every effort was made to yield data as cleanly as possible. However, if the system were to be used in the everyday management of patients, sequencing of uncloned, amplified products would be more practical and may yield comparable results. Specialty Laboratories (Santa Monica, CA, U.S.A.) is currently offering genotyping of heterogenous PCR product commercially. Alternatively, rapid sequencing of the polymerase and protease genes in development by Affymetrics (Sunnyvale, CA, U.S.A.) and Applied Biosystems (Foster City, CA, U.S.A.) using fluorescent primer-based cycle sequencing (29) may render the use of the CTSHIV expert system more feasible.

The CTSHIV system is limited by an incomplete understanding of the correlation between genomic mutations conferring drug resistance and clinical and surrogate marker outcomes. Furthermore, the expert program can only incorporate rules from the current literature and recommend licensed drugs. A study to correlate surrogate marker response and clinical outcomes with the use of the program is in progress. In addition, a study by the CPCRA is under way to assess the clinical utility of genotyping (Volberding $\mathrm{P}$, personal communication, February 1997). Studies with large numbers of patients will ultimately be required to validate the CTSHIV system and determine whether it may assist in the everyday management of HIV-infected individuals and in the design, implementation, and interpretation of outcomes in clinical trials (30). Currently, however, there is no evidence that the system will perform better than clinical judgment.

Acknowledgments: The technical assistance of Tonya Clark is appreciated. The authors thank Douglas Richman, M.D. for reviewing and revising the expert rules. The sequencing was performed by Erik Avajani and Clifford Brunk. This work was supported by the California Universitywide AIDS Research Program through the California Collaborative Treatment Group (CCTG).

\section{REFERENCES}

1. DeClercq E. Antiviral therapy for human immunodeficiency virus infections. Clin Microbiol Rev 1995;8:200-39.

2. Frost $S$, McLean $A$. Quasispecies dynamics and the emergence of drug resistance during zidovudine therapy of HIV infection. AIDS 1994;8:323-32.
3. Katlama C, Ingrand D, Loveday C, et al. Safety and efficacy of lamivudine-zidovudine combination therapy in antiretroviral-naive patients. JAMA 1996;276:118 25.

4. Holodniy M, Katzenstein D, Winters M, et al. Measurement of HIV virus load and genotypic resistance by gene amplification in asymptomatic subjects treated with combination therapy. $J$ Acquir Immune Defic Syndr 1993;6:366-9.

5. Kahn J, Lagakos S, Richman D, et al. A controlled trial comparing zidovudine with didanosine in human immunodeficiency virus infection: The NIAID AIDS Clinical Trials Group. $N$ Engl $J$ Med 1992;327:581 . 7 .

6. Schooley RT, Ramirez-Ronda C, Lange JM, et al. Virologic and immunologic benefits of initial combination therapy with zidovudine and zalcitabine or didanosine compared with zidovudine monotherapy: Wellcome Resistance Study Collaborative Group. $J$ Infect Dis 1996;173:1354-66.

7. Zhu QY, Scarborough A, Polsky B, Chou TC. Drug combinations and effect parameters of zidovudine, stavudine, and nevirapine in standardized drug-sensitive and resistant HIV type 1 strains. AIDS Res Hum Retrovinuses 1996;12:507-17.

8. Lange JM. Triple combinations: Present and future. $J$ Acquir Immune Defic Syndr Hum Retrovirol 1995;10(suppl 1):S77-82.

9. Nielsen C, Bruun L, Mathiesen L, Pedersen C, Gerstoft J. Development of resistance to zidovudine (ZDV) and didanosine (ddl) in HIV from patients in ZDV, ddl and alternating ZDV/ddl therapy. AIDS 1996;10:625-33.

10. Larder B, Kellam P, Kemp S. Zidovudine resistance predicted by direct detection of mutations in DNA from HIV-infected lymphocytes. AIDS 1991;5:137-44.

11. Larder B, Kemp S. Multiple mutations in HIV-1 reverse transcriptase confer high-level resistance to zidovudine (AZT). Science 1989;246:1155-8.

12. Frenkel L, Wagner M, Atwood S, Cummins T, Dewhurst S. Specific, sensitive, and rapid assay for HIV pol mutations associated with resistance to zidovudine and didanosine. J Infect Dis 1995; 33:342-7.

13. Jackson P. Introduction to expert systems. Reading, MA: AddisonWesley, 1990.

14. Pazzani M, Kibler D. The utility of knowledge in inductive learning. Machine learning 1992;9:57-94.

15. Kellam P, Boucher C, Larder BA. Fifth mutation in human immunodeficiency virus type 1 reverse transcriptase contributes to the development of high-level resistance to zidovudine. Proc Natl Acad Sci USA 1992;89:1934-8.

16. Fontenant $G$, Johnston $\mathrm{K}$, Cohen J, Gallaher W, Robinson J, Luftig R. PCR amplification of HIV-1 proteinase sequences directly from lab isolates allows determination of five conserved domains. Virol 1992;190: $1-10$

17. Gu Z, Gao Q, Fang H, et al. Identification of a mutation at codon 65 in the IKKK motif of reverse transcriptase that encodes human immunodeficiency virus resistance to $2^{\prime}, 3^{\prime}$-dideoxycytidine and 2', 3'-dideoxy-3'-thiacytidine. Antimicrob Agents Chemother 1994;38:275-81.

18. Clark J. Novel non-templated nucleotide addition reactions catalyzed by procaryotic and eucaryotic DNA polymerases. Nucleic Acid Res 1988;20:9677-86.

19. Giesecke H, Obermaier B, Domdey H. Rapid sequencing of the Sendai virus $6.8 \mathrm{~kb}$ large (L) gene through primer walking with an automated DNA sequencer. $J$ Virol Methods 1992;38:47-60.

20. St Clair M, Martin J, Tudor W. Resistance to ddl and sensitivity to AZT induced by a mutation in HIV-1 reverse transcriptase. Science 1991;253:1557-9.

21. Gu Z, Gao Q, Li X, Parniak M, Wainberg M. Novel mutation in the human immunodeficiency virus type 1 reverse transeriptase gene that encodes cross-resistance to $2^{\prime}, 3^{\prime}$-dideoxyinosine and $2^{\prime}, 3^{\prime}$ dideoxycytidine. J Virol 1992;66:7128-35.

22. Gu Z, Gao Q, Fang H, Parniak M, Brenner B, Wainberg M. Identification of novel mutations that confer drug resistance in the 
human immunodeficiency virus polymerase gene. Leukemia 1994; 8 SI:5166-9.

23. Richman D, Shih C, Lowy I, Rose J, Prodanovich P, Goff S, Griffin J. Human immunodeficiency virus type 1 mutants resistant to nonnucleoside inhibitors of reverse transcriptase arise in tissue culture. Proc Natl Acad Sci USA 1991;88:11241 5.

24. El-Farrash M, Kuroda M, Kitazaki T, Masuda T, Kato K, Hatanaka $M$, Harada S. Generation and characterization of a human immunodeficiency virus type 1 (HIV-1) mutant resistant to an HTV-1 protease inhibitor. $J$ Virol 1994;68:233-9.

25. Dueweke T, Pushkarskaya T, Poppe S, et al. A mutation in reverse transcriptase of bis(heteroaryl)piperazine-resistant human immunodeficiency virus type 1 that confers increased sensitivity to other non-nucleoside inhibitors. Proc Natl Acad Sci USA 1993;90: 4713-7.

26. Hammer S, Kessler H, Saag M. Issues in combination antiretroviral therapy: a review. J Acquir Immune Defic Syndr 1994;7 Suppl 2:\$24 35 .

27. Holodniy M, Mole L, Margolis D, et al. Determination of human immunodeficiency virus RNA in plasma and cellular viral DNA genotypic zidovudine resistance and viral load during zidovudinedidanosine combination therapy. $J$ Virol 1995;69:3510-6.

28. Michael N, Davis KE, Loomis-Price L, VanCott T, Burke D, Redfield R, Birx D. V3 seroreactivity and sequence variation: Tracking the emergence of V3 genotypic variation in HIV-1-infected patients. AIDS 1996;10:121-9.

29. Luehrsen KR, Marr L, van der Knaap E, Cumberledge S. Analysis of differential display RT-PCR products using fluorescent primers and GENESCAN software. Biotechniques 1997;22:168-74.

30. Ohno-Machado L, Parra E, Henry S, Tu S, Musen M. AIDS 2: A decision-support tool for decreasing physician's uncertainty regarding patient eligibility for HIV treatment protocols. Proceedings of the 17th Annual Symposium on Computer Applications in Medical Care, Washington, DC, 1993:429-33.

31. Boucher C, O'Sullivan E, Mulder A, et al. Ordered appearance of zidovudine resistance mutations during treatment of 18 human immunodeficiency virus-positive patients. J Infect Dis 1992;165: $105-10$.

32. Gurusinghe A, Land $\mathrm{S}$, Birch $\mathrm{C}$, et al. Reverse transcriptase mutations in sequential HIV-1 isolates in a patient with AIDS. $J$ Med Virol 1995;46:238-43.

33. Caliendo A, Savara A, An D, DeVore K, Kaplan J, D'Aquila R. Effects of zidovudine-selected human immunodeficiency virus type 1 reverse transcriptase amino acid substitutions on processive DNA synthesis and viral replication. $J$ Virol 1996;70:2146-53.

34. Quan Y, Gu Z, Li X, Li Z, Morrow C, Wainberg M. Endogenous reverse transcription assays reveal high-level resistance to the triphosphate of $(-) 2^{\prime}$-dideoxy-3'-thiacytidine by mutated M184V human immunodeficiency virus type $1 . J$ Virol 1996;70:5642-5.

35. Iversen AK, Shafer RW, Wearly K, Winters MA, Mullins JI, Chesebro B, Morgan TC. Multidrug-resistant human immunodeficiency virus type 1 strains resulting from combination antiretroviral therapy. I Virol 1996;70:1086-90.

36. Hooker D, Tachedjian G, Soloman A, et al. An in vivo mutation from leucine to tryptophan at position 210 in human immunode- ficiency virus type 1 reverse transcriptase contributes to high-level resistance to $3^{\prime}$-azido-3'-deoxythymidine. $J$ Virol 1996;70:8010-8.

37. Kew Y, Saloman H, Olsen L, Wainberg M. Prasad V. The nucleoside analog-resistant E89G mutant of human immunodeficiency virus type 1 reverse transeriptse displays a broader cross-resistance that cxtends to nonnuclcoside inhibitors. Antimicrob Agents Chemother 1996;40:1711-4.

38. Zhu Q, Scarborough A, Polsky B, Chou T. Drug combinations and effect parameters of zidovudine, stavudine, and nevirapine in standardized drug-sensitive and resistant HIV type 1 strains. AIDS Res Hum Retroviruses 1996;12:507-17.

39. Richman D, Havlir D, Corbeil J, et al. Nevirapine resistance mutations of human immunodeficiency virus type 1 selected during therapy. $J$ Virol 1994;68:1660-6.

40. Richman DD. Resistance of clinical isolates of human immunodeficiency virus to antiretroviral agents. Antimicrob Agents Chemother 1993;37:1207-13.

41. Richman D, Shih CK, Lowy I, Rose J, Prodanovich P, Goff S, Griffin J. Human immunodeficiency virus type 1 mutants resistant to nonnucleoside inhibitors of reverse transcriptase arise in tissue culture. Proc Natl Acad Sci USA 1991;88:11241-5.

42. Havlir, Eastman S, Gamst A, Richman D. Nevirapine-resistant human immnodeficiency virus: Kinetics of replication and estimated prevalence in untreated patients. $J$ Virol 1996;70:7894-9.

43. Dueweke TJ, Pushkarskaya T, Poppe SM, et al. A mutation in reverse transcriptase of bis(heteroaryl) piperazine-resistant human immunodeficiency virus type 1 that confers increased sensitivity to other non-nucleoside inhibitors. Proc Natl Acad Sci USA 1993;90: 4713-7.

44. Ho DD, Toyoshima $\mathrm{T}, \mathrm{Mo} \mathbf{H}$, et al. Characterization of human immunodeficiency virus type 1 variants with increased resistance to a C2-symmetic protease inhibitor. $J$ Virol 1994;68:2016-2020.

45. Kaplan AH, Michael SF, Wehbie RS, et al. Selection of multiple human immunodeficiency virus type 1 variants that encode viral proteases with decreased sensitivity to an inhibitor of viral protease. Proc Natl Acad Sci USA 1994;91:5597-601.

46. Boucher C. Rational approaches to resistance using saquinavir. AIDS 1996;10(suppl 1):S15-9.

47. Schmidt J, Ruiz L, Clotet B, et al. Resistance-related mutations in the HIV-1 protease gene of patients treated for 1 year with the protease inhibitor ritonavir (ABT-538). AIDS 1996;10:995-9.

48. Condra J, Holder D, Schlief W, et al. Genetic correlates of in vivo viral resistance to indinavir, a human immunodeficiency virus type 1 protease inhibitor. $J$ Virol 1996;70:8270-6.

49. Patick A, Duran M, Cao Y, et al. Genotypic analysis of HIV-1 variants isolated from patients treated with the protease inhihitor Nelfinavir, alone or in combination with $\mathrm{d} 4 \mathrm{~T}$ or AZT and 3TC [Abstract]. Presented at the Fourth Conference on Retroviruses and Opportunistic Infections, Washington, DC, 1997.

50. Jacobsen $\mathrm{H}$, Haenggi M, Ott M, Duncan I, Andreoni M, Vella S, Mous J. Reduced sensitivity to saquinavir: An update on genotyping from phase 1/II trials. Antiviral Res 1996;29:95-7.

51. Taburet A, Singlas E. Drug interactions with antiviral drugs. Clin Pharmacokinet 1996;30:385-401. 